\title{
Essentially-Euclidean convex bodies
}

\author{
by
Alexander E. Litvak (Edmonton), Vitali D. Milman (Tel Aviv) and Nicole TomczaK-JaEgermann (Edmonton)

\begin{abstract}
In this note we introduce a notion of essentially-Euclidean normed spaces (and convex bodies). Roughly speaking, an $n$-dimensional space is $\lambda$-essentially-Euclidean (with $0<\lambda<1$ ) if it has a $[\lambda n]$-dimensional subspace which has further proportionaldimensional Euclidean subspaces of any proportion. We consider a space $X_{1}=\left(\mathbb{R}^{n},\|\cdot\|_{1}\right)$ with the property that if a space $X_{2}=\left(\mathbb{R}^{n},\|\cdot\|_{2}\right)$ is "not too far" from $X_{1}$ then there exists a $[\lambda n]$-dimensional subspace $E \subset \mathbb{R}^{n}$ such that $E_{1}=\left(E,\|\cdot\|_{1}\right)$ and $E_{2}=\left(E,\|\cdot\|_{2}\right)$ are "very close." We then show that such an $X_{1}$ is $\lambda$-essentially-Euclidean (with $\lambda$ depending only on quantitative parameters measuring "closeness" of two normed spaces). This gives a very strong negative answer to an old question of the second named author. It also clarifies a previously obtained answer by Bourgain and Tzafriri. We prove a number of other results of a similar nature. Our work shows that, in a sense, most constructions of the asymptotic theory of normed spaces cannot be extended beyond essentially-Euclidean spaces.
\end{abstract}

1. Introduction. The asymptotic theory of normed spaces, as well as the asymptotic study of centrally symmetric convex bodies, has revolved mainly around properties of "Euclidean type." One searched for large sections well isomorphic to Euclidean spaces, for well complemented subspaces (which were often actually Euclidean) and so on. It was not necessarily the goal of the theory but a consequence of the methods used. For example, the following problem was formulated in [M3]:

Problem. Is it true that for every $\beta>0$ and $R>1+\beta$ there exists $a \lambda=\lambda(\beta, R) \in(0,1]$ such that for each $n$ and all $X_{1}=\left(\mathbb{R}^{n},\|\cdot\|_{1}\right)$ and $X_{2}=\left(\mathbb{R}^{n},\|\cdot\|_{2}\right)$ with $d\left(X_{1}, X_{2}\right)<R$, there exists a $k$-dimensional subspace $E \subset \mathbb{R}^{n}$ such that $k \geq \lambda n$ and $d\left(E_{1}, E_{2}\right) \leq 1+\beta$, where $E_{1}=\left(E,\|\cdot\|_{1}\right)$ and $E_{2}=\left(E,\|\cdot\|_{2}\right)$ ?

2010 Mathematics Subject Classification: 46B06, 52A23, 46B20.

Key words and phrases: convex bodies, Euclidean sections, essentially Euclidean convex bodies, Banach-Mazur distance, geometric distance, $M$-position, $M$-ellipsoid, covering numbers, projections. 
Clearly, if one space is Euclidean then the answer is affirmative (see, e.g., [M2]). Bourgain and Tzafriri gave a very strong negative answer in [BT] for spaces $\ell_{p}^{n}$ for $p>2$ and the Banach-Mazur distance. This shows that even a "nice" geometry of uniformly smooth and uniformly convex spheres does not ensure a positive answer. This example, however, is not easy, as it uses, in a very precise quantitative form, all accumulated knowledge about the structure of subspaces of $\ell_{p}^{n}$. A simpler (counter-)example is provided by $\ell_{\infty}^{n}$ by using results of [FJ] (we thank W. B. Johnson for this recent observation). This is based on rougher arguments but does not have any additional geometric properties which appeared implicitly in the background of [M3. So the question of how far constructions of the asymptotic theory can be extended beyond the "Euclidean" framework remained open.

We show in this note that, actually, the theory cannot be extended beyond "essentially-Euclidean" spaces. (For this and related definitions see Section 3 and note that these definitions involve a number of (fixed) parameters.) For example, we not only provide a negative answer to the problem above, but in fact we give a characterization of spaces under consideration by answering the following question.

Question. Let $\lambda \in(0,1], \beta>0$, and $R>1+\beta$; let $n$ be a positive integer. Assume that a space $X_{1}=\left(\mathbb{R}^{n},\|\cdot\|_{1}\right)$ has the property that whenever $X_{2}=\left(\mathbb{R}^{n},\|\cdot\|_{2}\right)$ is a space satisfying $d\left(X_{1}, X_{2}\right)<R$ then there exists $a[\lambda n]$-dimensional subspace $E \subset \mathbb{R}^{n}$ such that $d\left(E_{1}, E_{2}\right) \leq 1+\beta$, where $E_{1}=\left(E,\|\cdot\|_{1}\right), E_{2}=\left(E,\|\cdot\|_{2}\right)$. What can be said about $X_{1}$ ?

We show (in Theorem 4.3 and remarks following it) that if $X_{1}$ has such a property (in terms of the geometric distance) for a fixed triple $(\beta, R, \lambda)$ of parameters, with $\beta>0, R>2(1+\beta), \lambda \in(0,1]$, then $X_{1}$ is $\lambda$-essentiallyEuclidean. This means, roughly speaking, that $X_{1}$ has a $[\lambda n]$-dimensional subspace, which has further proportional-dimensional Euclidean subspaces of any proportion. This provides a strong negative answer to the problem discussed in two ways. Firstly, it characterizes a class of spaces $X_{1}$ for which the problem has a positive solution for every space $X_{2}$ such that the geometric distance $d\left(X_{1}, X_{2}\right)<R$. (Strictly speaking, Theorem 4.3 proves one implication only, but the converse implication is well-known.) Secondly, note that in the problem, given $\beta$ and $R>1+\beta, \lambda$ is a function of $\beta$ and $R$, while Theorem 4.3 and remarks afterwards provide the answer for an arbitrary choice of parameters $(\beta, R, \lambda)$ satisfying $R>2(1+\beta)$. It also explains why Bourgain-Tzafriri's example works only for $p>2$ (namely, as is well-known, for $p \leq 2$ the $\ell_{p}^{n}$ spaces are essentially-Euclidean; while for $p>2$ the $\ell_{p}^{n}$ spaces are not essentially-Euclidean, see Section 3). We would also like to mention that we do not know an answer for the case 
$1+\beta<R \leq 2(1+\beta)$. Although we might expect the answer to be the same we do not exclude a possibility of some different surprising result. Finally, in Section 5 we prove results of a similar nature relating the existence of nice proportional-dimensional sections to coverings of symmetric convex bodies.

2. Notation. We consider $\mathbb{R}^{n}$ with the standard Euclidean structure. The canonical Euclidean norm on $\mathbb{R}^{n}$ is denoted by $|\cdot|$, and the corresponding inner product by $\langle\cdot, \cdot\rangle$. The Euclidean unit ball and the Euclidean unit sphere are denoted by $B_{2}^{n}$ and $S^{n-1}$, respectively.

By a body we always mean a compact star-shaped body. By a symmetric body we mean a body that is centrally-symmetric with respect to the origin. Let $K$ be a convex body in $\mathbb{R}^{n}$ containing the origin. The polar body $K^{0}$ is given by

$$
K^{0}:=\left\{x \in \mathbb{R}^{n} \mid\langle x, y\rangle \leq 1 \text { for every } y \in K\right\} .
$$

We recall that for every subspace $E$ of $\mathbb{R}^{n}$ the polar (in $E$ ) of $K \cap E$ is $P_{E} K^{0}$, where $P_{E}$ is the orthogonal projection onto $E$.

Given a symmetric convex body $K \subset \mathbb{R}^{n}$ we will use the notation $\|\cdot\|_{K}$ for the Minkowski functional of $K$. The normed space $\left(\mathbb{R}^{n},\|\cdot\|_{K}\right)$ will also be denoted by $\left(\mathbb{R}^{n}, K\right)$. The geometric distance between $K \subset \mathbb{R}^{n}$ and $L \subset \mathbb{R}^{n}$ is defined by

$$
d_{g}(K, L):=\inf \{b / a \mid a>0, b>0, a K \subset L \subset b K\} .
$$

If $d_{g}(K, L) \leq C$ then we say that $K$ and $L$ are $C$-equivalent. The BanachMazur distance between $K$ and $L$ is defined by

$$
d(K, L):=\inf \left\{d_{g}(K, T L)\right\},
$$

where the infimum is taken over all invertible linear operators $T$ from $\mathbb{R}^{n}$ to $\mathbb{R}^{n}$. The Banach-Mazur distance between normed spaces is defined as the Banach-Mazur distance between their unit balls. If the Banach-Mazur distance between a space and the Euclidean space is bounded by $C$ we say that the space is $C$-Euclidean.

Let $K, L \subset \mathbb{R}^{n}$. The covering number $N(K, L)$ is the smallest integer $m$ such that $m$ shifts of $L$ cover $K$. If $K$ is a convex body, then a standard volume argument shows that $N(K, \varepsilon K) \leq(1+2 / \varepsilon)^{n}$.

The $n$-dimensional volume of a body $K$ in $\mathbb{R}^{n}$ is denoted by $|K|$.

Finally, we will need the definition of $M$-position of a convex body. This requires the following theorem of the second named author ([M4], see also Chapter 7 of [Pi]).

THEOREM 2.1. There exists an absolute constant $C$ such that for every $n \geq 1$ and every symmetric convex body $K \subset \mathbb{R}^{n}$ there exists a linear 
operator $T$ on $\mathbb{R}^{n}$ satisfying

$$
N\left(T K, B_{2}^{n}\right) \leq \exp (C n) \quad \text { and } \quad N\left(B_{2}^{n}, T K\right) \leq \exp (C n) .
$$

The image of $K$ under a linear operator $T$ satisfying Theorem 2.1 is called an $M$-position of $K$.

We will use the following property of $M$-position. In fact, this property played a role in the probabilistic proof of the quotient-of-subspace theorem by the second named author.

Proposition 2.2. Let $C>0$ and let $K \subset \mathbb{R}^{m}$ be a symmetric convex body such that

$$
N\left(K, B_{2}^{m}\right) \leq \exp (C m) .
$$

Then for every $0<\varepsilon<1$ there exists an $[\varepsilon m]$-dimensional subspace $E \subset \mathbb{R}^{m}$ such that

$$
K \cap E \subset C_{\varepsilon} B_{2}^{m} \cap E,
$$

where $C_{\varepsilon}$ depends only on $\varepsilon$ and $C$.

The proposition follows immediately from the volume ratio theorem ([ST]), used for example in the (equivalent) form of Proposition 5.1. It suffices to note that the Minkowski sum $K_{1}=K+B_{2}^{m}$ satisfies $N\left(K_{1}, 2 B_{2}^{m}\right) \leq$ $\exp (\mathrm{Cm})$ and $K_{1} \supset B_{2}^{m}$.

We shall also provide a direct elementary argument, for the convenience of the readers.

Proof. Without loss of generality we assume that $m \geq 3$. Let $r<1$ be a (small) positive parameter, which we specify later. Denote $N:=N\left(K, B_{2}^{m}\right)$ $=N\left(r K, r B_{2}^{m}\right) \leq \exp (C m)$. By the definition of covering numbers there exist $N$ points $x_{1}, \ldots, x_{N}$ in $\mathbb{R}^{m}$ such that

$$
r K \subset \bigcup_{i=1}^{N}\left(x_{i}+r B_{2}^{m}\right) \text {. }
$$

Let

$$
\bar{K}:=\left\{y \in S^{m-1}|| x-y \mid \leq r \text { for some } x \in(r K) \cap S^{m-1}\right\}
$$

and, for $i \leq N$, let

$$
S_{i}:=\left\{y \in S^{m-1}|| x-y \mid \leq r \text { for some } x \in\left(x_{i}+r B_{2}^{m}\right) \cap S^{m-1}\right\} .
$$

Then

$$
\bar{K} \subset \bigcup_{i=1}^{N} S_{i} .
$$

Clearly, every $S_{i}$ is a cap of radius at most (in the geodesic metric) $\alpha=$ $\arcsin (2 r)$. Let $\mu$ denote the normalized Lebesgue measure on $S^{m-1}$. Direct 
calculation (see, e.g., $\mathrm{MS}$ ) shows that

$$
\mu\left(S_{i}\right) \leq \frac{\int_{0}^{\alpha} \sin ^{m-2} t d t}{\int_{0}^{\pi} \sin ^{m-2} t d t} \leq \alpha \sin ^{m-2}(\alpha) \frac{\sqrt{m-2}}{2} \leq \sqrt{m-2}(2 r)^{m-1} .
$$

Thus

$$
\mu(\bar{K}) \leq e^{C m}(3 r)^{m-1} .
$$

Now let $F$ be a (fixed) $k$-dimensional subspace of $\mathbb{R}$ with $k=[\varepsilon m]$. Let $\mathcal{N}$ be an $r$-net (in the Euclidean metric) in $F \cap S^{m-1}$ of cardinality $|\mathcal{N}|<$ $(3 / r)^{k-1}$ (existence of such a net follows by a standard volume argument). Let $\mathbb{P}$ be the normalized Haar measure on the group $O(m)$ of orthogonal operators. Using the uniqueness of Haar measure and the union bound, we obtain

$$
\begin{aligned}
& \mathbb{P}(\{U \in O(m) \mid \exists a \in \mathcal{N} \text { such that } U a \in \bar{K}\}) \\
& \qquad \leq|\mathcal{N}| \mu(\bar{K})<(3 / r)^{\varepsilon m-1} e^{C m}(3 r)^{m-1}<1,
\end{aligned}
$$

provided that

$$
1 / r \geq\left(3^{1+\varepsilon} e^{2 C}\right)^{1 /(1-\varepsilon)} .
$$

Thus there exists an operator $U \in O(m)$ such that the subspace $E=U F$ satisfies $U a \notin \bar{K}$ for every $a \in \mathcal{N}$. Since $\{U a\}$ is an $r$-net in $E \cap S^{m-1}$ it follows that for every $x \in E \cap S^{m-1}$ we have $x \notin r K$, i.e.

$$
(r K) \cap E \subset B_{2}^{m} \cap E .
$$

The choice $r=\left(3 e^{C}\right)^{-2 /(1-\varepsilon)}$ implies the result.

Since $N\left(K \cap F, B_{2}^{n} \cap F\right) \leq N\left(K, B_{2}^{n}\right)$ for every subspace $F \subset \mathbb{R}^{n}$, the proposition immediately implies the following corollary.

Corollary 2.3. Let $K \subset \mathbb{R}^{n}$ be a symmetric convex body in $M$-position. Let $0<\delta<1$ and $F \subset \mathbb{R}^{n}$ be $a[\delta n]$-dimensional subspace. Then for every $0<\varepsilon<1$ there exists an $[\varepsilon \delta n]$-dimensional subspace $F_{0} \subset F$ such that

$$
K \cap F_{0} \subset C(\varepsilon, \delta) B_{2}^{n} \cap F_{0},
$$

where $C(\varepsilon, \delta)$ depends only on $\varepsilon$ and the ratio $C / \delta$ (where $C$ is the constant from the definition of an $M$-position). In particular, if for some $R>0$,

$$
B_{2}^{n} \cap F \subset R K \cap F,
$$

then

$$
\frac{1}{R} B_{2}^{n} \cap F_{0} \subset K \cap F_{0} \subset C(\varepsilon, \delta) B_{2}^{n} \cap F_{0} .
$$

REMARK. One can get a better dependence of $C(\varepsilon, \delta)$ on $\varepsilon$ and $\delta$ than the one that follows from the proof of Proposition 2.2 above using so-called regular $M$-position. Recall that a convex body $K$ is said to be in regular 
$M$-position if for every $t>0$,

$$
N\left(K, t B_{2}^{n}\right) \leq \exp \left(C_{0} n / t\right) \quad \text { and } \quad N\left(B_{2}^{n}, t K\right) \leq \exp \left(C_{0} n / t\right),
$$

where $C_{0}$ is an absolute positive constant independent of the dimension, $t$, and the body $K$. (This position is often called 1-regular, because the power of $t$ in the exponent equals to 1 . However, since in this paper we do not use other regular positions, we suppress the number 1 in the definition.) Actually, Pisier showed a more delicate fact that for any positive $\alpha<2$, the above bound for covering numbers can be replaced by $\exp \left(C_{\alpha} n / t^{\alpha}\right)$ (see e.g. [Pi, Chapter 7] for the proof). A strengthening to regular $M$-positions is not essential for Corollary 2.3. Moreover, we do not use this notion further on, except in the proof of Theorem 4.3 .

3. Essentially-Euclidean bodies. We introduce the following definitions.

Fix a function $d=d(\lambda, \varepsilon) \geq 1$ on $(0,1] \times(0,1)$. Let $\lambda \in(0,1]$. A body $K \subset \mathbb{R}^{n}$ is called $\lambda$-ess-Euclidean if there exists a $[\lambda n]$-dimensional section $K \cap F$ of $K$ such that for every $\varepsilon \in(0,1)$ there exists a further $[\varepsilon \lambda n]$ dimensional section of $K \cap F$ which is $d$-Euclidean. Note that this definition also depends on $d$, although this dependence is suppressed in the terminology. The case $\lambda=1$ is of importance and has appeared implicitly in many contexts. It contains, in particular, bodies with bounded volume ratios $([\mathrm{ST}])$, unit balls of cotype-2 spaces ([FLM], [M1], [DS]), unit balls of weak-cotype-2 spaces ([MP], see also $[\mathrm{Pi}])$.

Let $0<\delta \leq \lambda<1$ and $d \geq 1$. A body $K \subset \mathbb{R}^{n}$ is called $(\lambda, \delta)$-essEuclidean if for every $[\lambda n]$-dimensional section of $K$ there exists a further $[\delta n]$-dimensional section which is $d$-Euclidean.

We extend these notions to normed spaces in the standard way by saying that a space $X=\left(\mathbb{R}^{n},\|\cdot\|\right)$ is $\lambda$-ess-Euclidean (resp. $(\lambda, \delta)$-ess-Euclidean) if its unit ball is $\lambda$-ess-Euclidean (resp. $(\lambda, \delta)$-ess-Euclidean).

We would like to emphasize that, as usual in the asymptotic theory of normed spaces, for a fixed value of the parameter $\lambda$ and a fixed function $d=d(\varepsilon)$, we consider the class of $\lambda$-ess-Euclidean convex bodies in $\mathbb{R}^{n}$ for an arbitrary $n \geq 1$. Similarly, for fixed values of parameters $\lambda, \delta$ and $d$, we consider $(\lambda, \delta)$-ess-Euclidean convex bodies in $\mathbb{R}^{n}$, for all $n \geq 1$. In particular, all results below are stated for a given convex body in $\mathbb{R}^{n}$, and describe relevant parameters of the body and dependences between them in full detail, by formulas valid for all $n \geq 1$. Of course, if the dimension $n$ is small (say, $n<2 / \lambda$ or $n \leq d^{2}$ ) then any symmetric convex body is $\lambda$-ess-Euclidean and $(\lambda, \delta)$-ess-Euclidean, and so our results are really interesting in suitably high dimensions only. 
It should be noted that the terminology "essentially-Euclidean spaces/ bodies" freely used throughout the paper is rather a jargon of the asymptotic theory of normed spaces than a precise notion (in the same spirit as, for example, type- 2 and cotype- 2 spaces, spaces of bounded volume ratio, and many others). We believe that it provides the right intuition of the subject and at the same time is not misleading - especially that, as mentioned earlier, results below are stated in precise terms of all parameters involved.

Finally, we would like to recall that, given (fixed) $p>2$, the class of $\ell_{p}^{n}$ spaces, $n \geq 1$, is not a subset of the class of all $\lambda$-ess-Euclidean or $(\lambda, \delta)$ ess-Euclidean spaces (for any choice of parameters). Indeed, for $p=\infty$, it is well known that $\ell_{\infty}^{n}$ does not have Euclidean subspaces of dimension essentially higher than $\log n$. More precisely, if $E$ is a $k$-dimensional subspace of $\ell_{\infty}^{n}$ satisfying $d\left(E, \ell_{2}^{k}\right) \leq d$ then $k \leq C(d) \log n$, where $C(d)$ is a constant depending only on $d$ ([M2], see also 5.7 of [MS]). In fact, sharper estimates are known, namely, for such an $E$ one has $d\left(E, \ell_{2}^{k}\right)>c \sqrt{k / \log (n / k)}$, which implies that $k \leq C d^{2} \log \left(n / d^{2}\right)$, where $c, C$ are absolute positive constants (see [G]). For $2<p<\infty$, the space $\ell_{p}^{n}$ cannot have Euclidean subspaces of dimension higher than $C_{p} n^{2 / p}$. That is, if $F$ is a $k$-dimensional subspace of $\ell_{p}^{n}$ satisfying $d\left(E, \ell_{2}^{k}\right) \leq d$ then $k \leq d^{2} p n^{2 / p}$ ([BDGJN], see also 5.6 of [MS]). Thus, for large enough $n$, the space $\ell_{p}^{n}, 2<p \leq \infty$, cannot be $\lambda$-ess-Euclidean or $(\lambda, \delta)$-ess-Euclidean.

4. Lipschitz functions on convex bodies. In 1971 the second named author used properties of Lipschitz functions on the sphere and the concentration phenomenon on the sphere to provide a new proof of Dvoretzky's Theorem ([M2], see also [FLM] and [MS]). The new argument was much simpler than the original one and led to better (in fact, the best possible) estimates on the dimension of Euclidean sections of a convex body. It also provided a powerful general tool to study the behavior of high-dimensional convex bodies. In particular, the following stabilization result was proved ([M2], see also [MS, 2.4]).

TheOREM 4.1. Let $f: S^{n-1} \rightarrow \mathbb{R}^{+}$be a 1-Lipschitz function in the Euclidean metric and let $M_{f}$ be the median of $f$. Let $\varepsilon>0$ and set $\lambda=$ $\varepsilon^{2} /(2 \log (4 / \varepsilon))$. Then there exists a $[\lambda n]$-dimensional subspace $E \subset \mathbb{R}^{n}$ such that $\left|f(x)-M_{f}\right| \leq 2 \varepsilon$ for all $x \in S^{n-1} \cap E$.

In particular, under the hypotheses of the theorem, considering the cases $M_{f} \leq 6 \varepsilon$ and $M_{f}>6 \varepsilon$, it is clear that the subspace $E$ satisfies the (nonexclusive) dichotomy: either $f(x) \leq 8 \varepsilon$ for all $x \in S^{n-1} \cap E$, or $(2 / 3) M_{f} \leq$ $f(x) \leq(4 / 3) M_{f}$ for all $x \in S^{n-1} \cap E$. 
In this section we consider similar dichotomies for Lipschitz functions on a general convex body and show that they can hold if and only if the body is essentially-Euclidean.

Let $K \subset \mathbb{R}^{n}$ be a symmetric convex body. Let $0<\lambda<1$ and $\alpha, R>0$. We say that $K$ has property $L$ (with parameters $\lambda, \alpha, R$ ) if for every 1Lipschitz function $f$ (in the metric $\|\cdot\|_{K}$ ) defined on the boundary $\partial K$ of $K$, there exists a $[\lambda n]$-dimensional subspace $E \subset \mathbb{R}^{n}$ such that either $f(\cdot) \leq \alpha$ on $\partial K \cap E$ or $f(\cdot) \geq R$ on $\partial K \cap E$. It is noteworthy that in the latter case the lower bound assumed for $f$ already implies, as a byproduct of the argument, the stronger condition that $f$ is equivalent to $R$.

Note that property $L$ does not depend on the position of a body $K$, that is, if $K$ has property $L$ then so does $T K$ for any invertible linear operator $T$. Indeed, given a function $f$ on the boundary of $T K$ we can consider the function $g$ on the boundary of $K$ defined by $g(x)=f(T x)$ and apply property $L$ of $K$.

THEOREM 4.2. There exists an absolute constant $C$ such that the following holds. Let $n \geq 1$ and $K$ be a symmetric convex body in $\mathbb{R}^{n}$. Let $0<\lambda<1$ and $\alpha, R>0$. If $\alpha<1 /(4 \exp (C / \lambda))$ and $K$ has property $L$ with parameters $\lambda, \alpha$, and $R$ then $K$ is $\lambda$-ess-Euclidean with $d(\lambda, \varepsilon)<C(\varepsilon, \lambda) R$, where $C(\varepsilon, \lambda)$ depends only on $\varepsilon$ and the ratio $C / \lambda$.

Stabilization Theorem 4.1 says that $B_{2}^{n}$ has property $L$, and it is easy to see that if $K$ is essentially-Euclidean then it also has property $L$.

In a sense, Theorem 4.2 provides some kind of uniqueness: if a body $K$ has property $L$ then $K$ is essentially-Euclidean. So stabilization (and property $L$ which is weaker) can be achieved only by relating to some Euclidean structure.

Proof. Let $C$ be the constant from the definition of $M$-position and assume without loss of generality that $K$ is in $M$-position. Consider the body $B=\operatorname{conv}\left(K \cup B_{2}^{n}\right)$. Consider the function $f$ on $\partial K$ defined by $f(x)=\|x\|_{B}$. Clearly,

$$
|f(x)-f(y)| \leq\|x-y\|_{B} \leq\|x-y\|_{K},
$$

i.e., $f$ is 1 -Lipschitz. By property $L$ of $K$ there exists a $[\lambda n]$-dimensional subspace $E$ of $\mathbb{R}^{n}$ such that either $f(\cdot) \leq \alpha$ on $\partial K \cap E$ or $f(\cdot) \geq R$ on $\partial K \cap E$.

First we show that the former case is impossible, due to the assumption on $\alpha$. Indeed, $f(\cdot) \leq \alpha$ on $\partial K \cap E$ means $\|x\|_{B} \leq \alpha\|x\|_{K}$ on $E$, which implies

$$
K \cap E \subset \alpha\left(\operatorname{conv}\left(K \cup B_{2}^{n}\right)\right) \cap E \subset \alpha\left(K+B_{2}^{n}\right) \cap E .
$$

On the other hand, since $K$ is in $M$-position, we have

$$
N\left(K+B_{2}^{n}, 2 K\right) \leq N\left(B_{2}^{n}, K\right) \leq \exp (C n) .
$$


Using standard properties of covering numbers we obtain

$$
N\left(\left(K+B_{2}^{n}\right) \cap E, 4 K \cap E\right) \leq \exp (C n) .
$$

This yields

$$
N\left(\left(K+B_{2}^{n}\right) \cap E, 4 \alpha\left(K+B_{2}^{n}\right) \cap E\right) \leq \exp (C n),
$$

which is impossible by comparison of volumes and the condition on $\alpha$.

Thus we have $f(\cdot) \geq R$ on $\partial K \cap E$, that is, $\|x\|_{B} \geq R\|x\|_{K}$ on $E$. In particular,

$$
B_{2}^{n} \cap E \subset R K \cap E .
$$

Applying Corollary 2.3 we obtain the desired result.

We conclude this section with a theorem providing a negative answer to the problem stated in the Introduction. More precisely, this theorem gives a characterization of bodies that have the property in question by stating that only essentially-Euclidean bodies can have it. The proof is very similar to the proof of Theorem 4.2 and is provided for the sake of completeness.

Theorem 4.3. Let $\alpha \geq 1, R \geq 3 \alpha$, and $\lambda>0$. Assume that a symmetric convex body $K \subset \mathbb{R}^{n}$ has the property "if $d_{g}(K, L) \leq R$ for a symmetric convex body $L \subset \mathbb{R}^{n}$ then there exists a subspace $E \subset \mathbb{R}^{n}$ of dimension at least $\lambda n$ and such that $d_{g}(K \cap E, L \cap E) \leq \alpha$." Then $K$ is $\lambda$-ess-Euclidean with

$$
d(\lambda, \varepsilon)<C_{1} C(\varepsilon, \lambda) R\left(\lambda \log \frac{2 R}{5 \alpha}\right)^{-1},
$$

where $C(\varepsilon, \lambda)$ depends only on $\varepsilon, \lambda$, and $C_{1}$ is an absolute positive constant.

REMARK 1 . One can check that in fact our proof works for every $R>2 \alpha$ and gives

$$
d(\lambda, \varepsilon)<C_{1} C(\varepsilon, \lambda) \frac{\alpha R}{R-2 \alpha}\left(\lambda \log \frac{2 R}{R+2 \alpha}\right)^{-1} .
$$

Remark 2. Note that Theorem 4.3 answers the question stated in the Introduction.

Proof. Without loss of generality we can assume that $K$ is in regular $M$-position, that is, for every $t>0$,

$$
N\left(K, t B_{2}^{n}\right) \leq \exp \left(C_{0} n / t\right) \quad \text { and } \quad N\left(B_{2}^{n}, t K\right) \leq \exp \left(C_{0} n / t\right)
$$

(see the remark following Corollary 2.3).

Set $r=\frac{\lambda}{5 C_{0}} \log \frac{2 R}{5 \alpha}$ and consider the following two bodies:

$$
S:=\operatorname{conv}\left(K \cup r B_{2}^{n}\right) \quad \text { and } \quad L:=S \cap R K .
$$

Clearly, $K \subset L \subset R K$. Thus, by the property of the body $K$, there exists a subspace $E \subset \mathbb{R}^{n}$ of dimension $k \geq \lambda n$ and such that $d_{g}(K \cap E, L \cap E) \leq \alpha$. 
This means that there is $0<a \leq R$ such that

$$
\frac{a}{\alpha} K \cap E \subset L \cap E \subset a K \cap E .
$$

First we show that $a<R$. Assume that $a=R$. Then, clearly,

$$
|L \cap E| \geq\left(\frac{R}{\alpha}\right)^{k}|K \cap E| .
$$

On the other hand,

$$
\begin{aligned}
N(L \cap E,(5 / 2) K \cap E) \leq N(L,(5 / 4) K) \leq N\left(K+r B_{2}^{n},(5 / 4) K\right) \\
\leq N\left(r B_{2}^{n},(1 / 4) K\right)=N\left(B_{2}^{n},(1 / 4 r) K\right) \leq \exp \left(4 C_{0} r n\right) .
\end{aligned}
$$

Therefore,

$$
|L \cap E| \leq\left(\frac{5}{2}\right)^{k} \exp \left(4 C_{0} r n\right)|K \cap E|
$$

This yields

$$
\frac{R}{\alpha} \leq \frac{5}{2} \exp \left(4 C_{0} r(n / k)\right)
$$

which contradicts our choice of $r$ (recall that $k \geq \lambda n$ ).

Now observe that since $L \cap E \subset a K \cap E$ and $a<R$, we have

$$
S \cap E \subset a K \cap E .
$$

Indeed, let $x \in S \cap E$. If $x \in R K$ then $x \in L \cap E$ and the inclusion holds. If $x \notin R K$ then $\|x\|_{K}>R$. Define $y=R x /\|x\|_{K}$. Then $\|y\|_{K}=R, y \in S \cap E$, $y \in R K$. Hence $y \in L \cap E \subset a K \cap E$. Thus $\|y\|_{K} \leq a$. This contradicts the fact $\|y\|_{K}=R>a$.

The inclusions $S \cap E \subset a K \cap E \subset R K \cap E$ imply

$$
\frac{r}{R} B_{2}^{n} \cap E \subset K \cap E,
$$

and the proof is completed by invoking Corollary 2.3 .

5. Properties "from coverings to sections". We now introduce properties of convex bodies which we call "from coverings to sections" and denote by $\mathbf{c} / \mathbf{s}$. They relate the covering number of an arbitrary convex body by a given convex body to the geometric distance between some sections of these bodies. There will be two underlying parameters $A>0$ and $R>1$, related to the covering and the distance, respectively, which we usually omit in our notation.

Our results will use the following well-known immediate corollary of the volume ratio theorem $([\mathrm{ST}]$, see also $[\mathrm{Pi}$, Chapter 6$])$.

Proposition 5.1. Let $K \subset \mathbb{R}^{m}$ be a convex body and $\mathcal{E}$ be an ellipsoid such that

$$
\mathcal{E} \subset K \quad \text { and } \quad N(K, c \mathcal{E}) \leq C^{m}
$$


for some constants $C, c \geq 1$. Then for every $k \leq m$ there exists a $k$ dimensional subspace $E \subset \mathbb{R}^{m}$ such that

$$
\mathcal{E} \cap E \subset K \cap E \subset d \mathcal{E} \cap E,
$$

where

$$
d \leq(4 \pi c C)^{m /(m-k)} .
$$

Proof. The hypothesis clearly yields $|K| /|\mathcal{E}| \leq(c C)^{m}$, hence the result follows from the volume ratio theorem.

Remark. Of course, Proposition 2.2 immediately implies Proposition 5.1, with worse dependence of the distance $d$ on the parameters $c$, $C$, and $m /(m-k)$.

5.1. Property $\mathbf{c} / \mathbf{s} 1$. We define property $\mathbf{c} / \mathbf{s} 1$ as follows.

Let $A>0$ and $R>1$. Let $0<\delta \leq \lambda<1$. We say that a body $K \subset \mathbb{R}^{n}$ has property $(\lambda, \delta)$-c/s1 if for every body $L \supset K$ satisfying $N(L, K) \leq \exp (A n)$ the following holds. For every $[\lambda n]$-dimensional $E \subset \mathbb{R}^{n}$ there exists a $[\delta n]$ dimensional $F \subset E$ such that $L \cap F \subset R K \cap F$.

Note that this property does not depend on the position of the body $K$, that is, if $K$ has c/s1 then so does $T K$ for any invertible linear operator $T$.

TheORem 5.2. Let $K$ be a symmetric convex body in $\mathbb{R}^{n}$. Let $A \geq C$ $+\log 5$ and $R>1$, where $C$ is the constant from the definition of $M$ position. Let $0<\delta \leq \lambda<1$. If $K$ has $(\lambda, \delta)$-c/s1 then $K$ is $(\lambda, \varepsilon \delta)$-essEuclidean for every $\varepsilon \in(0,1)$. Conversely, if $K$ is $(\lambda, \delta)$-ess-Euclidean then $K$ has $(\lambda, \varepsilon \delta)$-c/s1 for every $\varepsilon \in(0,1)$.

REMARK 1. The second part of the theorem is known. We provide a proof for completeness.

REMARK 2. The proof below gives in the first part $d \leq C(\varepsilon, \delta) R$, where $C(\varepsilon, \delta)$ is the constant from Corollary 2.3, and in the second part $R \leq$ $(8 \pi d \exp (A / \delta))^{1 /(1-\varepsilon)}$.

Proof. Without loss of generality assume that $K$ is in $M$-position. Consider the body $L=\operatorname{conv}\left(K \cup B_{2}^{n}\right)$. Then

$$
N(L, K) \leq N\left(K+B_{2}^{n}, K+K\right) N(2 K, K) \leq \exp (C n) 5^{n} \leq \exp (A n) .
$$

Observe that by property $\mathbf{c} / \mathbf{s} \mathbf{1}$, for every $[\lambda n]$-dimensional $E \subset \mathbb{R}^{n}$ there exists a $[\delta n]$-dimensional $F \subset E$ such that $L \cap F \subset R K \cap F$, which implies

$$
B_{2}^{n} \cap F \subset R K \cap F \text {. }
$$

Applying Corollary 2.3, we obtain

$$
\frac{1}{R} B_{2}^{n} \cap F_{0} \subset K \cap F_{0} \subset C(\varepsilon, \delta) B_{2}^{n} \cap F_{0} .
$$

This implies the result with $d=C(\varepsilon, \delta) R$. 
To prove the second part of the theorem we assume that $K$ is $(\lambda, \delta)$-essEuclidean and that $L \supset K$ satisfies $N(L, K) \leq \exp (A n)$. By the definition, for every $[\lambda n]$-dimensional $E \subset \mathbb{R}^{n}$ there exists a $[\delta n]$-dimensional $F \subset E$ such that

$$
\mathcal{E} \cap F \subset K \cap F \subset d \mathcal{E} \cap F,
$$

for some ellipsoid $\mathcal{E}$. Then

$$
N(L \cap F, 2 d \mathcal{E} \cap F) \leq N(L \cap F, 2 K \cap F) \leq N(L, K) \leq \exp (A n)
$$

and $\mathcal{E} \cap F \subset L \cap F$. Proposition 5.1 applied to $L \cap F$ and the inclusion $\mathcal{E} \cap F \subset K \cap F$ imply the desired result with

$$
R=(8 \pi d \exp (A / \delta))^{1 /(1-\varepsilon)} .
$$

5.2. Property $\mathbf{c} / \mathbf{s 2}$. Another property from coverings to sections, $\mathbf{c} / \mathbf{s} \mathbf{2}$, is similar but slightly stronger than property $\mathbf{c} / \mathbf{s} \mathbf{1}$, as the requirement $L \supset K$ is dropped.

Let $A>0$ and $R>1$. Let $0<\delta \leq \lambda<1$. We say that a body $K \subset \mathbb{R}^{n}$ has property $(\lambda, \delta)$-c/s2 if for every body $L$ satisfying $N(L, K) \leq \exp (A n)$ the following holds. For every $[\lambda n]$-dimensional $E \subset \mathbb{R}^{n}$ there exists a $[\delta n]$ dimensional $F \subset E$ such that $L \cap F \subset R K \cap F$.

As before, $\mathbf{c} / \mathbf{s} \mathbf{2}$ does not depend on the position of a body $K$.

The relation between property $\mathbf{c / s 2}$ and being essentially-Euclidean is the same as for property $\mathbf{c} / \mathbf{s} 1$ in Theorem 5.2 , but in this case the restriction on $A$ becomes weaker (or actually unnecessary).

Theorem 5.3. Let $K$ be a symmetric convex body in $\mathbb{R}^{n}$. Let $A \geq C$ and $R>1$, where $C$ is the constant from the definition of $M$-position. Let $0<\delta \leq \lambda<1$. If $K$ has $(\lambda, \delta)$-c/s2 then $K$ is $(\lambda, \varepsilon \delta)$-ess-Euclidean for every $\varepsilon \in(0,1)$. Conversely, if $K$ is $(\lambda, \delta)$-ess-Euclidean then $K$ has $(\lambda, \varepsilon \delta)$-c/s2 for every $\varepsilon \in(0,1)$.

The proof repeats the argument of Theorem 5.2. The only difference is that we consider $L=B_{2}^{n}$ instead of $L=\operatorname{conv}\left(K \cup B_{2}^{n}\right)$ and adjust the covering estimates. Moreover, using regular $M$-position (see the proof of Theorem 4.3) one can prove the theorem for every $A>0$.

5.3. Property c/s3. We define one more property of the c/s-type, called $\mathbf{c} / \mathbf{s} 3$. In the definition below we identify $\mathbb{R}^{n}$ with the coordinate subspace of $\mathbb{R}^{N}, N \geq n$, of vectors having zeros in the last $N-n$ coordinates.

Let $A>0$ and $R>1$. Let $0<\lambda<1$. We say that a body $K \subset \mathbb{R}^{n}$ has property $\lambda$-c/s3 if for every $N$, every body $L \subset \mathbb{R}^{N}$ satisfying $K=L \cap \mathbb{R}^{n}$ and $N(P L, K) \leq \exp (A n)$ for some projection $P$ from $\mathbb{R}^{N}$ onto $\mathbb{R}^{n}$ the 
following holds. There exists a $[\lambda n]$-dimensional subspace $E \subset \mathbb{R}^{n}$ such that $(P L) \cap E \subset R K \cap E$.

REMARK. Let $L \subset \mathbb{R}^{N}$ be a body satisfying the two conditions above. Clearly the projection $P$ does not need to have a well bounded norm (in the norm induced by $L$ ). However, in the presence of property $\lambda$-c/s3, there exists an $F \subset \mathbb{R}^{N}$ of dimension $N-n+[\lambda n]$, that contains $E$ and such that there exists a bounded projection $Q: F \rightarrow E$. Indeed, take $F=\operatorname{ker} P \oplus E$ and $Q=P_{\mid F}$. Then, since $E \subset \mathbb{R}^{n}=\operatorname{Im} P$, we have

$$
\operatorname{dim} F=\operatorname{dim} \operatorname{ker} P+\operatorname{dim} E=N-n+[\lambda n]
$$

and

$$
Q(L \cap F)=P(L \cap F)=(P(L \cap F)) \cap E \subset R K \cap E=R L \cap E,
$$

which means $\|Q:(F, L \cap F) \rightarrow(E, L \cap E)\| \leq R$.

TheOREM 5.4. Let $K$ be a symmetric convex body in $\mathbb{R}^{n}$. Let $A \geq C+$ $\log 5$ and $R>1$, where $C$ is the constant from the definition of $M$-position. Let $0<\lambda<1$. If $K$ has $\lambda$-c/s3 then $K$ is $\lambda$-ess-Euclidean. Conversely, if $K$ contains an $[\lambda n]$-dimensional section which is $d$-Euclidean, then $K$ has $(\varepsilon \lambda)-\mathbf{c} / \mathbf{s} 3$ for every $\varepsilon \in(0,1)$.

Proof. Let $N=2 n$. Let $\mathcal{B} \subset \mathbb{R}^{n}$ be an $M$-ellipsoid for $K$. Define the ellipsoid $\mathcal{E} \subset \mathbb{R}^{N}$ by

$$
\mathcal{E}=\{(x, x) \mid x \in \mathcal{B}\} .
$$

Let $L$ be the convex hull of $K \cup \mathcal{E}$ and $P$ be the orthogonal projection on $\mathbb{R}^{n}$. Then $L \cap \mathbb{R}^{n}=K$ and $P L=\operatorname{conv}(K \cup \mathcal{B})$. As in the proof of Theorem 5.2 we obtain

$$
N(P L, K) \leq \exp (A n)
$$

By property $\mathbf{c} / \mathbf{s} 3$ it follows that there exists a $[\lambda n]$-dimensional $E \subset \mathbb{R}^{n}$ such that $(P L) \cap E \subset R K \cap E$. Thus $\mathcal{B} \cap E \subset R K \cap E$. Since $\mathcal{B}$ is an $M$-ellipsoid for $K$, applying Corollary 2.3 we find that for every $\varepsilon \in(0,1)$ there exists a further $[\varepsilon \lambda n]$-dimensional subspace $E_{1}$ of $E$ such that

$$
\frac{1}{R} \mathcal{B} \cap E_{1} \subset K \cap E_{1} \subset C(\varepsilon, \lambda) \mathcal{B} \cap E_{1} .
$$

Thus $K \cap E_{1}$ is $C(\varepsilon, \lambda) R$-Euclidean, which shows that $K$ is $\lambda$-ess-Euclidean with $d=C(\varepsilon, \lambda) R$.

The proof of the second part is similar to the proof of Theorem 5.2 . Assume that for a $[\lambda n]$-dimensional $E \subset \mathbb{R}^{n}$ we have $\mathcal{E} \subset K \cap E \subset d \mathcal{E}$ for some ellipsoid $\mathcal{E} \subset E$. Assume that a body $L \subset \mathbb{R}^{N}$ and a projection $P$ from 
$\mathbb{R}^{N}$ onto $\mathbb{R}^{n}$ satisfy $L \cap \mathbb{R}^{n}=K$ and $N(P L, K) \leq e^{A n}$. Then

$$
N((P L) \cap E, 2 d \mathcal{E}) \leq e^{A n} .
$$

By Proposition 5.1 applied to $(P L) \cap E$ we infer the existence of an $[\varepsilon \lambda n]$ dimensional subspace $F \subset E$ such that

$$
(P L) \cap F \subset(8 \pi d \exp (A / \lambda))^{1 /(1-\varepsilon)} \mathcal{E} .
$$

Since $\mathcal{E} \subset K \cap E$, the result follows.

Acknowledgments. V. D. Milman was partially supported by BSF grant No. 2006079 and ISF grant No. 387/09. N. Tomczak-Jaegermann holds the Canada Research Chair in Geometric Analysis.

\section{References}

[BDGJN] G. Bennett, L. E. Dor, V. Goodman, W. B. Johnson and C. M. Newman, On uncomplemented subspaces of $L_{p}, 1<p<2$, Israel J. Math. 26 (1977), 178-187.

[BT] J. Bourgain and L. Tzafriri, Embedding $l_{p}^{k}$ in subspaces of $L_{p}$ for $p>2$, ibid. 72 (1990), 321-340.

[DS] S. J. Dilworth and S. J. Szarek, The cotype constant and an almost Euclidean decomposition for finite dimensional normed spaces, ibid. 52 (1985), 82-96.

[FJ] T. Figiel and W. B. Johnson, Large subspaces of $l_{\infty}^{n}$ and estimates of the Gordon-Lewis constant, ibid. 37 (1980), 92-112.

[FLM] T. Figiel, J. Lindenstrauss and V. D. Milman, The dimension of almost spherical sections of convex bodies, Acta Math. 139 (1977), 53-94.

[G] E. D. Gluskin, Extremal properties of orthogonal parallelepipeds and their applications to the geometry of Banach spaces, Mat. Sb. (N.S.) 136 (1988), 85-96 (in Russian); English transl.: Math. USSR-Sb. 64 (1989), 85-96.

[M1] V. D. Milman, Random subspaces of proportional dimension of finite-dimensional normed spaces: approach through the isoperimetric inequality, in: Banach Spaces (Columbia, MO, 1984), Lecture Notes in Math. 1166, Springer, Berlin, 1985, 106-115.

[M2] - A new proof of A. Dvoretzky's theorem on cross-sections of convex bodies, Funkcional. Anal. i Priložen. 5 (1971), no. 4, 28-37 (in Russian).

[M3] - Geometrical inequalities and mixed volumes in the local theory of Banach spaces, in: Colloquium in honor of Laurent Schwartz, Vol. 1 (Palaiseau, 1983), Astérisque 131 (1985), 373-400.

[M4] - An inverse form of the Brunn-Minkowski inequality, with applications to the local theory of normed spaces, C. R. Acad. Sci. Paris Sér. I Math. 302 (1986), 25-28.

[MP] V. D. Milman and G. Pisier, Banach spaces with a weak cotype 2 property, Israel J. Math. 54 (1986), 139-158.

[MS] V. D. Milman and G. Schechtman, Asymptotic Theory of Finite-Dimensional Normed Spaces, with an appendix by M. Gromov, Lecture Notes in Math. 1200, Springer, Berlin, 1986.

[Pi] G. Pisier, The Volume of Convex Bodies and Banach Space Geometry, Cambridge Univ. Press, Cambridge, 1989. 
[ST] S. J. Szarek and N. Tomczak-Jaegermann, On nearly Euclidean decomposition for some classes of Banach spaces, Compos. Math. 40 (1980), 367-385.

Alexander E. Litvak

Department of Mathematical

and Statistical Sciences

University of Alberta

Edmonton, Alberta, Canada T6G 2G1

E-mail: alexandr@math.ualberta.ca

Nicole Tomczak-Jaegermann

Department of Mathematical

and Statistical Sciences

University of Alberta

Edmonton, Alberta, Canada T6G 2G1

E-mail: nicole.tomczak@ualberta.ca
Vitali D. Milman

Department of Mathematics Raymond and Beverly Sackler Faculty of Exact Sciences Tel Aviv University, Tel Aviv, Israel E-mail: milman@post.tau.ac.il

Received October 14, 2008

Revised version October 21, 2009 